\title{
CCC trigrams of low association value: A re-evaluation'
}

ARTHUR F. COSTANTINI, DEPARTMENT OF PSYCHOLOGY, LYNDON STATE COLLEGE, Lyndon Center, Vt. AND RALPH O. BLACKWOOD, SCHOOL OF EDUCATION, UNIVERSITY OF AKRON, Akron, Ohio

The 343 CCC trigrams having the lowest association values in Witmer's 1935 list were re-evaluated. Reliability of ratings was 89 and the correlation with Witmer's values was.79. Large changes were found in trigrams such as JFK, QJF, LBJ, LBH, HFC, GXM, $D H J, D X B$, etc. There were substantial increases in values of trigrams beginning with $D$. New norms are presented for experimenters who need more precise control over stimulus materials.

Investigators of short-term memory, paired-associate learning and other processes, often use low association value CCC trigrams as their stimulus material (Peterson \& Peterson, 1959; Bruce \& Cofer, 1965). Since the only available association values for CCC trigrams are those published by Witmer in 1935, the investigator who wishes to obtain precise control over association values encounters difficulties. While it may be true that considerable stability in association value, meaningfulness, frequency, etc., has been found when words or CVC trigrams were studied (Goss \& Nodine, 1965; Underwood \& Schulz, 1960; Spreen \& Schulz, 1966), a casual inspection of Witmer's list produces obvious evidence of striking changes which have occurred over the last 30 years, suggesting that trigrams may not be as stable as some other verbal materials. For example, 30 years ago, under a different president, $71 \%$ of Witmer's Ss could think of an association for FDR but only $29 \%$ found LBJ meaningful. Before football and communist subversion became so highly organized, only $25 \%$ of Ss reported associations for NFL and in days when the financing of homes and a certain company's automobiles may have been less frequent, HFC and GXM had only $4 \%$ association values.

An investigator may reduce the variability in meaningfulness of his stimulus materials by eliminating trigrams which have obviously changed in association value, but this intuitive approach cannot lead to the elimination of trigrams which have changed in value due to more subtle associations. If investigators in short-term memory, or other areas, are to have available stimulus materials of uniform low association value, it seems essential to establish new norms.

The present study was undertaken to identify changes that have occurred over the past three decades and to make available to present investigators the new norms for low association-value CCC trigrams.

Method. The Ss were 80 students enrolled in introductory psychology classes at the University of Bridgeport. The Ss were divided into eight groups of approximately 20 Ss per group. Each group participated in one experimental session which consisted of rating 200 trigrams.

The 343 trigrams having the lowest association values in Witmer's list ( 0 to $17 \%)$ were selected for re-evaluation. The rating method which Noble, Stockwell, \& Pryor (1957) introduced was employed. Each trigram was flashed upon a screen for $5 \mathrm{sec}$ with an opaque projector. Ss were instructed to rate each trigram using a 5-point scale, indicating how many associations, ideas, or things it made them think of. The 5-point scale, printed at the top of each sheet, was as follows: $1=$ none, $2=$ below average, $3=$ average, $4=$ above average, $5=$ very many. Ss were directed to use the following procedure: (a) Write down the trigram, (b) decide which category on the 5-point scale was most appropriate for the trigram, (c) write down the number of that category next to the trigram, (d) cover the rated trigram and its rating with a mask (provided to reduce sequence effects) and prepare for the next trigram. Three practice examples were given and Ss were permitted to ask questions.

In order to assess the reliability of the ratings, 50 trigrams were repeated in the list, bringing the total list of trigrams to 393.
In previous studies of syllables, randomization has been limited to the counterbalancing of pages, the order of trigrams on each page remaining the same throughout. A number of investigators have expressed dissatisfaction with this procedure (Loess \& Nodine, 1965), suggesting that order effects may influence the ratings of some trigrams. Therefore, to control for the occurrence of order effects four different random lists of the 393 trigrams were produced by an IBM 1620 computer, using a 3-digit random number generator. Each of the four lists was divided in half and each half was given to a group of approximately $20 \mathrm{Ss}$ for rating. Thus, each of the 393 trigrams received ratings from about $80 \mathrm{Ss}$.

Results and Discussion. A reliability correlation of .89 was found between ratings of the 50 trigrams which were repeated in each list, indicating that the rating method was reliable. A Pearson Produce Moment correlation of .79 was found between Witmer's association values and those obtained in the present study. This value seems reasonably high if we consider that the distribution of association values was drastically truncated by selecting a narrow range of values.

As expected, individual trigrams have changed in association value over the past 30 years. For example, HFC, which received a higher rating than $98.8 \%$ of the 343 trigrams in the present study, received a rating higher than only $6.7 \%$ of the same 343 trigrams in the Witmer study. GXM received a rating higher than $6.9 \%$ of the 343 low association value trigrams in the Witmer study and $72.8 \%$ of the same trigrams in the present study.

Some changes seemed related to the presence of meaningful consonant pairs. Trigrams with any combination of two consonants from JFK or LBJ showed striking increases in association value (e.g., QJF, BFK, FSJ, LBH, and HBJ).

Other trends were found for which the explanations are not so obvious. For example, there were substantial increases in association values for those trigrams beginning with the letter $D$.

The results indicate that important changes have occurred in the association values of CCC trigrams over the past 30 years. Investigators who need more precise control over association value of CCC trigrams may wish to re-evaluate trigrams or to select trigrams from the new norms which are presented here.

Table 1 (on the next page) presents the 343 trigrams which were selected from Witmer's (1935) list $(0$ to $17 \%$ association values). The trigrams are listed here in rank order from lowest association value to highest association value, as rated in the present study.

\section{REFERENCES}

BRUCE, D., \& COFER, C. N. A comparison of recognition and recall in short-term memory. Proc. Amer. Psychol. Ason., 1965, 81-82.

GOSS, A. E., \& NODINE. C. F. Paired-associates learning: The role of meaningfulness, similarity, and familiarization. New York: Academic Press, 1965.

NOBLE, C. E., STOCKWELL, F. E., \& PRYOR, M. W. Meaningfulness $\left(m^{\prime}\right)$ and association value $(a)$ in paired-associate syllable learning. Psychol Rep., 1957, 3, 441-452.

PETERSON, L. R., \& PETERSON, M. J. Short-term retention of individual verbal items. J. exp. Psychol, 1959, 58, 193-198.

SPREEN, O., \& SCHULZ, R. W. Parameters of abstraction, meaningfulness, and pronunciability for 329 nouns. J. verbal Leam. verbal Behav., 1966, S, $459-468$.

UNDERWOOD, B. J., \& SCHULZ, R. W. Meaningfulness and verbal learning: Philadelphia: Lippincott, 1960. Lippincott, 1960.

WITMER, L. R. The association-value of three place consonant syllables. J. genet. Psychol, 1935, 47, 337-360.

\section{NOTE}

1. This research was supported in part by USPHS Grant MH 11017-01 and a University of Bridgeport Faculty Grant. 
Table 1

New Norms for Low Association-value CCC Trigrams

\begin{tabular}{|c|c|c|c|c|}
\hline 0 to $20 \%$ & $21 \%$ to $40 \%$ & $41 \%$ to $60 \%$ & $61 \%$ to $80 \%$ & $81 \%$ to $100 \%$ \\
\hline CGQ & ZJH & FPW & KXC & MJF \\
\hline XKQ & ZSJ & GCQ & QDJ & MWB \\
\hline JQZ & ZHW & KHQ & XHM & MGQ \\
\hline XQG & ZGF & FWQ & ZBQ & JQF \\
\hline HXJ & $\mathrm{FQJ}$ & ZQW & WZQ & $\mathrm{CWH}$ \\
\hline $\mathrm{ZQC}$ & KZF & KFQ & CFQ & SJF \\
\hline GCZ & ZXH & FCQ & QMW & MHB \\
\hline ZJX & $\mathrm{ZMX}$ & ZGX & QBJ & TFJ \\
\hline HXQ & HXXB & $\mathrm{XNZ}$ & TZQ & XBJ \\
\hline ZXJ & MHZ & $\mathrm{CXZ}$ & ZXR & DHU \\
\hline GZQ & $\mathrm{XQF}$ & GQC & GKQ & DJZ \\
\hline XZQ & HXF & ZTK & ZFW & ZBF \\
\hline QXH & ZHX & $\mathrm{DQH}$ & $\mathrm{CJZ}$ & BHJ \\
\hline XBN & CXQ & QJS & HFJ & MHJ \\
\hline XsZ & QXF & CJQ & $\mathrm{CZF}$ & DJH \\
\hline ZXC & ZKH & QGZ & BQF & JXF \\
\hline GXZ & ZJT & BQZ & MZB & JDW \\
\hline QXJ & KZS & GCJ & WCQ & JDF \\
\hline ZQJ & KZX & $\mathrm{XFH}$ & $\mathbf{J X B}$ & DJB \\
\hline$Q C X$ & ZQH & $\mathrm{CQZ}$ & TZF & FPJ \\
\hline XZK & KZG & $\mathrm{FQC}$ & KQW & DJS \\
\hline ZCK & $\mathrm{QFZ}$ & FJZ & $\mathrm{XQB}$ & MFH \\
\hline CXQ & QWJ & FXQ & $\mathrm{MQB}$ & MBX \\
\hline KZH & XFQ & ZTF & $\mathrm{XBF}$ & HВM \\
\hline ZXF & GXK & $\mathrm{KQH}$ & ZBJ & HJC \\
\hline XQD & KXQ & $\mathrm{KQZ}$ & $\mathrm{KXN}$ & LJF \\
\hline ZXD & TZH & QJF & QJC & JSB \\
\hline ZJQ & ZJF & ZDH & KXB & WBF \\
\hline ZWJ & XFZ & $\mathrm{CZJ}$ & XTG & BWF \\
\hline XZJ & GXZ & QCF & KHB & KBF \\
\hline ZSQ & ZTX & MWZ & BJQ & DJW \\
\hline $\mathrm{XZF}$ & XKH & ZQG & JQW & JDH \\
\hline $\operatorname{cs} X$ & ZDW & QFH & SBZ & MCF \\
\hline QGJ & GXC & QZB & FJH & XDJ \\
\hline XGZ & $\mathrm{JQH}$ & FJQ & KHF & JHW \\
\hline ZGW & $\mathrm{CXJ}$ & WQH & MWB & MPH \\
\hline GZK & JXQ & $\mathrm{ZJC}$ & $\mathrm{QHF}$ & LHJ \\
\hline XTJ & ZFJ & JXH & QXL & HBJ \\
\hline XZC & XGC & ZLC & XDH & HWC \\
\hline ZWQ & $\mathrm{FQH}$ & $\mathrm{ZQF}$ & DJQ & HFK \\
\hline GXJ & $\mathrm{QHJ}$ & XFP & TFQ & MWF \\
\hline ZTF & $\mathrm{QZH}$ & JTQ & XJF & JBP \\
\hline GQK & $\mathrm{ZQB}$ & QFC & GXM & WBH \\
\hline $\mathbf{X Q J}$ & KQX & QDX & HKM & JFM \\
\hline GCX & SZI & HZW & QHN & JFQ \\
\hline GKX & XGK & XJF & CHJ & JCF \\
\hline XBQ & CQK & MZJ & WBQ & BJH \\
\hline ZKG & $\mathrm{KXZ}$ & QFX & XKB & BPJ \\
\hline $\mathrm{QZJ}$ & XZD & GQB & CXF & JHC \\
\hline QLJ & GQH & KXD & XFK & JHF \\
\hline ZQS & KWQ & ZFK & LJX & WFC \\
\hline QGC & XFJ & QXM & GZB & JFC \\
\hline $\mathrm{XGQ}$ & HJX & KXR & DXJ & JFN \\
\hline ZXB & $\mathrm{XCJ}$ & $\mathrm{HCZ}$ & FJC & HFM \\
\hline $\mathrm{XQF}$ & QGW & XGP & KMF & BFM \\
\hline $\mathrm{XQH}$ & CXK & XHF & MHW & JFH \\
\hline XQL & CQJ & ZBG & TJW & LCF \\
\hline $\mathrm{zCX}$ & GJX & JZH & FJS & FPC \\
\hline ZHK & $\mathrm{ZQX}$ & KFX & GJC & BFM \\
\hline QKB & KQM & $\mathrm{XJB}$ & FHJ & WCF \\
\hline $\mathrm{CQJ}$ & $\mathrm{KXH}$ & $\mathrm{XJH}$ & GCJ & KBF \\
\hline XJQ & $\mathrm{XMZ}$ & ZFC & FJW & MPW \\
\hline $\mathrm{ZHJ}$ & CXF & ZBH & $\mathrm{HJF}$ & WBJ \\
\hline $\mathrm{ZCJ}$ & BQJ & CGJ & MHF & FHC \\
\hline ZXG & ZXK & FCJ & KBH & BMF \\
\hline XHJ & MQJ & $\mathrm{KQB}$ & FWJ & HFC \\
\hline ZFQ & QZM & XFS & SBQ & \\
\hline FQW & KHZ & FQK & $\mathrm{ZMC}$ & \\
\hline QSJ & QJF & HCJ & CFJ & \\
\hline
\end{tabular}

